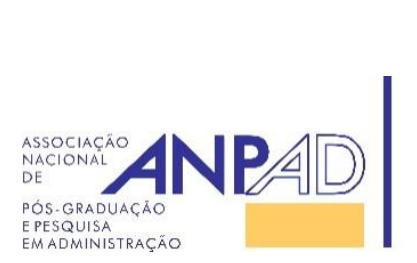
Disponível em
http://www.anpad.org.br/rac
RAC, Rio de Janeiro, v. 21, Edição Especial FCG, art. 1, pp. 1-21, Abril 2017

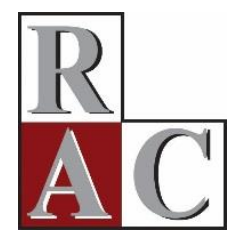

\title{
Avaliação de Desempenho no Governo Mineiro: Alterações dos Indicadores e Metas
}

Performance Evaluation in the Minas Government: Analysis of Changes of Indicators and Targets

\author{
Rosimeire Pimentel Gonzaga ${ }^{1}$ \\ Fabio Frezatti ${ }^{2}$ \\ Ivan Beck Ckagnazaroff ${ }^{1}$ \\ Janilson Antônio da Silva Suzart ${ }^{3}$ \\ Universidade Federal de Minas Gerais ${ }^{1}$ \\ Universidade de São Paulo ${ }^{2}$ \\ Secretaria do Tesouro Nacional ${ }^{3}$
}

Artigo recebido em 09.12.2015. Última versão recebida em 29.06.2016. Aprovado em 03.07.2016. Publicado online em 26.09.2016. 


\title{
Resumo
}

O modelo de gestão pública adotado pelo Estado de Minas Gerais, conhecido como Choque de Gestão, foi implementado utilizando-se de um instrumento de pactuação de resultados, denominado Acordo de Resultados, que prevê possíveis recompensas para o alcance das metas pactuadas. Considerando o Acordo de Resultados e a premissa de que os agentes fazem ajustes ou mudanças nos indicadores de desempenho nele contidos para adequar interesses distintos daqueles inicialmente almejados, esta pesquisa buscou identificar os fatores organizacionais e ambientais que influenciam as alterações dos indicadores de desempenho na estrutura gerencial do governo mineiro, à luz da teoria de agência e da teoria da contingência. Para alcançar o objetivo proposto, realizou-se um estudo de caso contendo abordagem qualitativa, utilizando-se de análise documental, questionário e entrevistas semiestruturadas para a coleta de dados. As entrevistas realizadas foram analisadas por meio de análise de conteúdo. Os resultados encontrados evidenciam que as ferramentas gerenciais aportadas no Choque de Gestão apresentam-se insuficientes para blindar a estrutura gerencial do modelo de gestão diante de algumas pressões identificadas com base nas teorias citadas, desvirtuando o modelo inicialmente proposto e promovendo alterações nos indicadores.

Palavras-chave: avaliação de desempenho organizacional; indicadores de desempenho; gestão por resultados; gestão pública; choque de gestão do Governo de Minas Gerais.

\begin{abstract}
The government of the Brazilian state of Minas Gerais adopted a management model, known as Management Shock, by way of covenants called Agreement on Results, which foresee possible rewards for the achievement of agreed upon targets. Considering such covenants and the assumption that agents can promote adjustments or changes in performance indicators contained therein in order to pursue interests different than those initially agreed upon, this research sought to identify the organizational and environmental factors influencing changes of performance indicators in the government's management structure. This research was conducted in light of the Agency and Contingency Theories. To this end, we conducted a qualitative case study, collecting data through documentary analysis, questionnaires and semi-structured interviews. The interviews were subsequently submitted to content analysis. The results show that the tools provided are not sufficient to shield the model's managerial structure from pressures identified using Agency and Contingency Theories. As a consequence the model as originally proposed was undermined, allowing for changes to indicators.
\end{abstract}

Key words: organizational performance evaluation; performance indicators; management by results; public management; Minas Gerais state Government management shock. 


\section{Introdução}

A gestão pública por resultados foi um dos principais elementos contidos no receituário da $\mathrm{New}$ Public Management, ou Nova Gestão Pública (doravante, NPM). A mesma se fundamenta na utilização de ferramentas gerenciais, como planejamento estratégico e indicadores para a avaliação de desempenho (Boland \& Fowler, 2000) e pode ser definida como o ciclo que se inicia com o estabelecimento das metas e dos resultados desejados, prossegue com a definição das medidas utilizadas para o monitoramento e a avaliação de desempenho, e finaliza com a retroalimentação do sistema com os resultados das avaliações (Gomes, 2009).

Em Minas Gerais, a partir da transição da gestão do Estado entre os governadores Itamar Franco e Aécio Neves, em 2002, implantou-se na gestão pública estadual o Choque de Gestão, contendo uma série de medidas para solucionar os problemas relacionados aos quadros econômico, fiscal e administrativo-institucional (Queiroz \& Ckagnazaroff, 2010; Silveira, Fonseca, \& Oliveira, 2013). O Estado apresentava grave situação financeira, com déficits fiscais, endividamento crescente e ausência de recursos para cobrir as despesas orçamentárias (Silveira \& Borges, 2008). Para a implementação do Choque de Gestão, foi utilizado um instrumento de avaliação de desempenho contendo indicadores denominado Acordo de Resultados, definido como um instrumento de pactuação de resultados entre o Poder Executivo e os servidores (Duarte, Lemos, Marini, \& Martins, 2006), com o intuito de aumentar a eficiência.

O monitoramento e a avaliação do desempenho apoiaram-se em indicadores a serem alcançados pelos órgãos estaduais, estabelecendo-se como incentivo para seu alcance a possibilidade de recebimento de bonificação financeira (Borges \& Matias, 2011). O Acordo de Resultados começou a ser implementado pela Secretaria de Planejamento e Gestão (Seplag). Posteriormente, com a ajuda de uma consultoria externa, criaram-se os indicadores, que foram negociados caso a caso com as demais secretarias (Gomes, 2009). Contudo, alguns deles evoluíram e foram alterados com o decorrer do tempo.

Nesse aspecto da mudança de indicadores, autores como Jackson e Lapsley (2003), Bjørnenak, (1997) e Malmi (1999) têm-se dedicado a discussões envolvendo o processo de inovação e alternância na utilização de ferramentas gerenciais e indicadores de desempenho no setor privado e, sobretudo, no setor público, emergindo daí questionamentos quanto aos fatores que influenciam essas alterações. Bjørnenak (1997) identificou que variáveis relacionadas à estrutura organizacional, como tamanho, influenciam as mudanças realizadas em metas e indicadores utilizados para avaliar o desempenho das empresas que atuam no setor privado, e ressaltou a importância da influência de fatores institucionais sobre essas alterações.

No setor público, Jackson e Lapsley (2003) constataram que, além da influência do setor privado, fatores como regulação, recomendação de autoridades locais e agências governamentais influenciam as alterações da estrutura gerencial das organizações. Lapsley e Wright (2004) pesquisaram os possíveis motivos que conduzem ao abandono das ferramentas contábeis utilizadas na gestão pública, o que os autores consideraram como inovações contábeis nesse setor, apresentando os seguintes fatores: novas técnicas, custo na obtenção de informação, falhas das técnicas e perda de utilidade, e fatores não identificados ou sem resposta. Os autores argumentam, ainda, que as alterações na estrutura contábil no setor público ocorrem, em grande parte, por influência governamental (Lapsley \& Wright, 2004).

No que se refere aos indicadores utilizados para mensurar o desempenho, problemas no conteúdo ou nas dimensões dos indicadores formulados podem ser alguns dos fatores que levem às mudanças dos indicadores e fazer com que os agentes tomem ações diferentes daquelas requeridas pela instituição, como manipulação dos resultados, redução de esforços após o alcance do nível de resultado pactuado e foco em um conjunto restrito de atividades ligadas aos indicadores, desconsiderando as demais. Ainda nesse sentido, a alteração ou variabilidade dos indicadores utilizados para garantir o alcance das metas pode dificultar a comparabilidade entre indicadores capazes de oferecer algum subsídio para a avaliação da qualidade de determinada gestão (Hopwood, 1972). 
Assim, com base na literatura sobre indicadores de desempenho, na teoria da contingência e na teoria de agência, este trabalho se propõe a responder a seguinte questão de pesquisa: Quais são os fatores organizacionais ou ambientais que estão associados às alterações das metas e dos indicadores utilizados na avaliação de desempenho na estrutura gerencial do governo do Estado de Minas Gerais, na percepção dos servidores públicos?

O objetivo principal consiste em identificar os fatores organizacionais e ambientais que, na percepção dos servidores públicos, levam às alterações dos indicadores na estrutura gerencial do governo do Estado de Minas Gerais, com base em um estudo de caso.

Este estudo busca contribuir com a literatura sobre indicadores de desempenho, ao analisar, na estrutura gerencial do governo do Estado de Minas Gerais, os fatores ligados às mudanças das metas e dos indicadores utilizados, uma vez que informações sobre a existência e a evidenciação de indicadores utilizados para a avaliação de desempenho auxiliam no controle social e podem tornar mais simplificadas as análises de informações sobre o desempenho estatal em complemento a peças técnicas, como demonstrações contábeis e relatórios do setor público brasileiro.

Ainda, as metas e os indicadores, por meio da vinculação entre as estratégias estabelecidas e o planejamento e execução delas, cumprem o papel de fornecer informações para a avaliação sobre a economia, eficiência, eficácia e efetividade do gasto público (Borges \& Matias, 2011). Assim, entender como ocorrem os processos de escolha e evolução dessas metas e indicadores pode influenciar a qualidade dos serviços e das informações reportados para a população e tais dados podem ser utilizados na formulação de novas políticas públicas, além de auxiliar órgãos de controle, tais como Controladorias, Auditorias e Tribunais de Contas, nos processos de avaliações da execução de políticas públicas.

\section{Plataforma Teórica}

\section{A avaliação de desempenho e a nova gestão pública}

O processo de avaliação de desempenho pode ser entendido como o levantamento sistemático de dados e sua comparação com padrões previamente definidos, visando auxiliar o processo decisório, bem como avaliar a eficácia (fins alcançados) e a eficiência (economicidade dos meios) (Holanda, 2003). Seus modelos têm como principal função fornecer a seus usuários informações acerca do desempenho de determinada unidade, além de promover o alinhamento entre estratégias, metas, ações e incentivos oferecidos aos agentes.

Especificamente em relação à gestão pública, a avaliação de desempenho atua como suporte para instrumentos de prestação de contas e processos de aprendizagem para melhor o desempenho (Castaldelli \& Aquino, 2011). Guimarães (2008) ressalta que avaliação de desempenho tem um papel fundamental, estruturado em quatro etapas: (a) Diagnóstico inicial - atua na definição de diretrizes, a partir do cenário encontrado inicialmente; (b) Processo decisório - contribui para definir as estratégias de ação e avaliar a relação custo-benefício das estratégias adotadas; (c) Implementação de ações trabalha com o monitoramento dos resultados obtidos, considerando os ajustes necessários, em razão das mudanças de cenário; e (d) Finalização - realiza avaliações por meio de comparações entre os resultados obtidos e os planejados inicialmente.

Os modelos de avaliação de desempenho são associados a instrumentos contratuais ou semicontratuais entre agentes ou órgãos de determinada organização pública, atuando como instrumento para o estabelecimento de metas e objetivos, e, posteriormente, a critérios para medir e acompanhar os resultados gerados (Guimarães, 2008). Segundo Holanda (2003), podem ser considerados como objetos de instrumentos contratuais de avaliação na gestão pública: produtos, pessoas, desempenho, projetos, programas e políticas. 
Uma das visões mais importantes relacionadas à utilização da avaliação de desempenho na gestão pública foi introduzida pela literatura envolvendo a nova gestão pública (Brignall \& Modell, 2000). A nova gestão pública ou new public management (NPM) é a expressão utilizada por Christopher Hood para descrever os resultados encontrados a partir da reforma gerencial do setor público (Kurunmaki, Lapsley, \& Melina, 2003).

Uma das principais características da NPM é a utilização no setor público de elementos predominantemente do setor privado (Fryer, Antony, \& Ogden, 2009; Hood, 1991). A forma de atuação da gestão baseada na NPM se divide em quatro dimensões: descentralização da gestão; estabelecimento de contratos de avaliação de desempenho atrelado a incentivos gerenciais; terceirização de parte dos serviços públicos; e criação de taxas para os serviços públicos (Ayee, 2005). Segundo Jackson e Lapsley (2003), um dos principais elementos utilizados pela NPM para a gestão pública é o estabelecimento de indicadores a serem utilizados para avaliação de desempenho.

Nessa linha, integrantes do sistema de controle gerencial, os indicadores utilizados na avaliação de desempenho podem ser baseados em diversas medidas. Para Malina e Selto (2004), pesquisas referentes à escolha de medidas para mensurar o desempenho pelas entidades baseiam-se na dicotomia entre medidas financeiras e medidas não financeiras.

Na Tabela 1 apresentada, Jannuzzi (2005) elenca as doze principais propriedades que os indicadores devem, na medida do possível, possuir.

Tabela 1

\section{Propriedades Requeridas dos Indicadores no Setor Público}

\begin{tabular}{|c|c|c|}
\hline $\mathbf{N}^{\circ}$ & Propriedade & Justificativa \\
\hline 1 & Relevância & É necessário que o indicador seja relevante para a agenda política. \\
\hline 2 & Validade de representação & $\begin{array}{l}\text { O indicador deve estar o mais próximo possível da demanda política que } \\
\text { o originou. }\end{array}$ \\
\hline 3 & Confiabilidade da medida & Propriedade necessária à legitimação do indicador. \\
\hline 4 & Cobertura & $\begin{array}{l}\text { É necessário que a cobertura do indicador seja representativa da } \\
\text { realidade empírica em análise. }\end{array}$ \\
\hline 5 & Sensibilidade às ações previstas & É importante que sejam dispostas medidas sensíveis e específicas às \\
\hline 6 & Especificidade & $\begin{array}{l}\text { ações do objeto avaliado, para a avaliação dos efeitos de possíveis } \\
\text { intervenções. }\end{array}$ \\
\hline 7 & $\begin{array}{l}\text { Transparência metodológica na } \\
\text { sua construção }\end{array}$ & Propriedade necessária à legitimação técnica e científica do indicador. \\
\hline 8 & Comunicabilidade & $\begin{array}{l}\text { Importante para a garantia da transparência das decisões tomadas pelos } \\
\text { gestores públicos. }\end{array}$ \\
\hline 9 & $\begin{array}{l}\text { Factibilidade operacional para } \\
\text { sua obtenção }\end{array}$ & $\begin{array}{l}\text { Referente à relação custo-benefício de construção e utilização do } \\
\text { indicador. }\end{array}$ \\
\hline 10 & Periodicidade & $\begin{array}{l}\text { É necessário que seja mantida regularidade na atualização dos } \\
\text { indicadores nos períodos analisados. }\end{array}$ \\
\hline 11 & Desagregabilidade & $\begin{array}{l}\text { Relacionada ao foco dos indicadores. Os indicadores devem ser } \\
\text { implementados para a avaliação de objetos restritos, estratificando } \\
\text { grupos e tratamentos de suas características específicas. }\end{array}$ \\
\hline 12 & $\begin{array}{l}\text { Comparabilidade da série } \\
\text { histórica }\end{array}$ & $\begin{array}{l}\text { Permite a inferência de tendências e a avaliação dos efeitos de mudanças } \\
\text { implementadas. }\end{array}$ \\
\hline
\end{tabular}

Nota. Adaptada de Jannuzzi, P. D. M. (2002). Considerações sobre o uso, mau uso e abuso dos indicadores sociais na formulação e avaliação de políticas públicas municipais. Revista de Administração Pública, 36(1), 51-72; Jannuzzi, P. D. M. (2005). Indicadores para diagnóstico, monitoramento e avaliação de programas sociais no Brasil. Revista do Serviço Público Brasília, 56(2), 137-160. 
Ainda, para o alcance dos objetivos estratégicos nos diversos níveis organizacionais, além da observância às propriedades requeridas dos indicadores, é necessário maior entendimento sobre a classificação, a importância e a forma de utilização dos indicadores empregados na avaliação de desempenho (Fryer et al., 2009; Jannuzzi, 2005).

A escolha dos indicadores pode ser guiada pelas classificações dos mesmos. Podem ser encontrados na literatura gerencial trabalhos que oferecem uma taxonomia dos indicadores a serem utilizados e que supõem que algumas das dimensões demonstradas na Tabela 2 compõem os indicadores utilizados para avaliação de desempenho.

Tabela 2

Taxonomia dos Indicadores Utilizados para Avaliação de Desempenho

\begin{tabular}{|c|c|c|}
\hline Dimensão & Classificação & Autores \\
\hline \multirow[t]{2}{*}{ Natureza objetiva/subjetiva } & Objetiva & Jannuzzi (2005) \\
\hline & Subjetiva & \\
\hline \multirow[t]{5}{*}{ Natureza processual } & Indicador-insumo & Barzelay (1997) \\
\hline & Indicador-processo & Boland e Fowler (2000) \\
\hline & & Jannuzzi $(2002,2005)$ \\
\hline & maicador-produto ou inaicador-resuitado & Johnsen (2005) \\
\hline & Indicador-impacto & \\
\hline \multirow[t]{5}{*}{ Dimensão do desempenho } & Economicidade & Barzelay (1997) \\
\hline & Eficiência & Boland e Fowler (2000) \\
\hline & Fficácia & Johnsen (2005) \\
\hline & Eileatid & Castaldelli e Aquino (2011) \\
\hline & Efetividade & \\
\hline \multirow[t]{2}{*}{ Natureza analítico/sintética } & Analítica & Jannuzzi (2005) \\
\hline & Sintética & \\
\hline \multirow[t]{8}{*}{ Natureza dos atributos } & Autonomia & Barzelay (1997) \\
\hline & Relevância & Grateron (1999) \\
\hline & Pertinência & Julnes e Holzer (2001) \\
\hline & & Castaldelli e Aquino (2011) \\
\hline & Objetividade & \\
\hline & Sensibilidade & \\
\hline & Precisão & \\
\hline & Relação custo-benefício & \\
\hline
\end{tabular}

Nota. Elaborada pelos autores.

Os indicadores utilizados na avaliação de desempenho podem ser assim classificados:

- Quanto à sua natureza: objetivos ou subjetivos. Os primeiros referem-se a fatos concretos e são constituídos a partir de estatísticas ou base de dados disponíveis. Os últimos compreendem medidas estabelecidas por meio da avaliação de indivíduos com relação a diferentes aspectos da realidade, como percepção sobre o desempenho de determinado governo (Jannuzzi, 2005).

- Quanto à dimensão processual: indicador-insumo, indicador-processo, indicador-resultado e indicador-impacto (Boland \& Fowler, 2000; Jannuzzi, 2005; Johnsen, 2005). Nesse modelo, os indicadores-insumo podem ser considerados como recursos de entrada, sendo sempre quantificáveis, podendo ser físicos, humanos ou financeiros, como gasto com professores para cada aluno (Boland 
\& Fowler, 2000; Jannuzzi, 2005; Johnsen, 2005). Os indicadores-produto, ou indicadores-resultado, estão relacionados às saídas ou aos objetivos finais do objeto avaliado, podendo ser medidos em termos quantificáveis, como taxa de mortalidade infantil (Jannuzzi, 2005). Os indicadores-impacto estão relacionados aos efeitos e desdobramentos gerados em função da implantação de determinado programa, como melhoria na aprendizagem infantil, decorrente de melhoria no atendimento a gestantes (Jannuzzi, 2002, 2005). Os indicadores-processo são intermediários e têm como foco o esforço operacional de alocação de recursos (indicadores-insumo) para a obtenção de melhorias (indicadores-resultado e indicadores-impacto), como número de consultas médicas em determinado período (Barzelay, 1997; Boland \& Fowler, 2000; Jannuzzi, 2005; Johnsen, 2005).

Quanto à dimensão de desempenho: economicidade (ou economia), eficiência, eficácia e efetividade (Boland \& Fowler, 2000). A economia reflete a forma como a organização utiliza seus recursos e se relaciona com a eliminação de perdas nas entradas do processo, podendo ser observada pelas alterações ocorridas nos recursos de entrada ou dos indicadores-insumo (Boland \& Fowler, 2000; Johnsen, 2005). A eficiência é definida pela otimização do processo de transformação das entradas e saídas, estando focada na compreensão da medida em que as saídas atendem às necessidades organizacionais (Barzelay, 1997; Boland \& Fowler, 2000; Johnsen, 2005). A eficácia pode ser definida pela relação entre os objetivos de determinados programas e os resultados alcançados, representados pelas saídas ou indicadores-resultado. A efetividade é representada pelo impacto gerado pelos resultados gerados pelos processos (Barzelay, 1997; Johnsen, 2005).

Quanto à natureza analítica ou sintética dos indicadores: sintética - síntese de um conjunto de indicadores; e analítica - maior nível de detalhamento na construção do indicador (Jannuzzi, 2005).

Quanto à natureza dos atributos: autonomia, relevância, pertinência, objetividade, sensibilidade, precisão e relação custo-benefício (Barzelay, 1997; Julnes \& Holzer, 2001). A autonomia se refere ao fato de os indicadores serem ou não regulados (Julnes \& Holzer, 2001). A relevância está relacionada à acurácia do conteúdo dos indicadores. A pertinência se refere ao ajuste do indicador ao desempenho que está sendo avaliado. A objetividade apresenta a magnitude do valor para não permitir julgamento de valor. A sensibilidade foca o poder informacional do indicador em relação às possíveis variações. A precisão está embasada no fato de que uma margem de erro inerente ao indicador não pode produzir informações distorcidas. A relação custo-benefício estabelece que o custo de obtenção da informação gerada por determinado indicador não deve ultrapassar o benefício gerado (Castaldelli \& Aquino, 2011; Grateron, 1999).

\section{Teoria da contingência e teoria de agência}

Pesquisas baseadas na teoria da contingência buscam identificar a influência dos fatores contingentes sobre as organizações, considerando que eles influenciam a relação entre as características organizacionais e o desempenho (Donaldson, 2001).

A dinâmica da teoria da contingência envolve três categorias de análises relacionadas a: estrutura, desempenho e fatores contingentes (Marques, 2012). Podem ser classificados como fatores contingentes quaisquer variáveis que possuam um efeito moderador sobre a relação entre determinada característica organizacional e o desempenho da organização; ou seja, fatores que podem afetar a relação entre a estrutura e o desempenho organizacional (Donaldson, 2001).

A teoria da contingência trata, também, de processos de mudanças organizacionais, ponderando que as influências de variáveis ambientais sobre a organização proporcionam mudanças graduais em sua estrutura (Wright, Kroll, \& Parnell, 1998/2009). Segundo Naranjo-Gil (2009), a influência de fatores contingenciais é que define se as organizações adotam ou não mudanças em suas estruturas.

Donaldson (2001) acrescenta que o modelo principal analisado sob a ótica da teoria da contingência baseia-se no fato de que as estruturas organizacionais das instituições estão associadas às contingências e de que há adequação ou ajuste de algum nível da estrutura organizacional a cada nível 
de contingência capaz de provocar o aumento de desempenho ou desajuste com a redução do desempenho.

Estudos baseados na teoria da contingência têm constatado que as organizações respondem às mudanças por meio da adaptação e que, como resultado, elaboram ou modificam suas estruturas internas, a fim de manterem uma relação isomórfica com o ambiente (Astley \& Van de Ven, 2005). Assim, a teoria da contingência é utilizada para analisar a forma como fatores contingenciais, internos e externos, podem influenciar os processos de mudanças nas organizações (Otley, 1980; Upping \& Oliver, 2011). Os fatores externos são aqueles relacionados às incertezas quanto ao ambiente organizacional, à pressão de mercado e às questões políticas; já os internos são aqueles relacionados diretamente à estrutura organizacional, como tamanho, controle orçamentário e sistema de mensuração de desempenho (Otley, 1980; Upping \& Oliver, 2011).

Especificamente na gestão pública, alguns autores, como Lüder (1992), Christensen e Yoshimi (2003) e Upping e Oliver (2011) têm se dedicado a verificar as mudanças ocorridas nas organizações públicas, à luz da teoria da contingência.

Lüder (1992) analisou as mudanças ocorridas na estrutura contábil das organizações públicas por meio de um estudo comparativo entre Estados Unidos, Canadá e alguns países europeus, classificando os fatores capazes de influenciar as mudanças em: estimulantes - situação financeira, escândalo financeiro, mudança de padrão externo e interesse profissional; estruturais para usuários das informações - status socioeconômico e cultura política; características do sistema administrativopolítico para os produtores de informações - cultura administrativa, competição política, políticas de treinamento e recrutamento; e barreiras de implementação - características organizacionais, sistema legal, nível de qualificação e tamanho da jurisdição. Os resultados encontrados sugerem que o sucesso da implementação de um sistema contábil é influenciado pela combinação de fatores favoráveis e desfavoráveis às mudanças.

Baseando-se no modelo proposto por Lüder (1992), Christensen e Yoshimi (2003) buscaram identificar os fatores contingenciais relacionados às mudanças ocorridas no sistema contábil de um estado australiano. Os autores concluíram que as mudanças podem ser influenciadas por interesses dos agentes internos da organização e desestimuladas por fatores considerados como barreiras à implementação das mudanças.

Upping e Oliver (2011), por sua vez, modificaram o modelo proposto por Lüder (1992) ao analisarem mudanças ocorridas na estrutura contábil de universidades na Tailândia. Os resultados encontrados indicam que as mudanças ocorridas foram influenciadas por fatores relacionados a pressões externas, pressões internas e facilitadores das mudanças.

Ainda considerando os fatores que podem influenciar mudanças na estrutura envolvida na gestão pública, segundo autores como Jensen e Meckling (1976), a teoria de agência pode ser utilizada para explicar os fenômenos ocorridos na gestão pública, uma vez que considera a relação contratual entre o agente e principal no setor público, na qual existem diferentes níveis informacionais, sendo necessário prever elevados custos para a obtenção das informações ou a quebra da assimetria, o que pode resultar no problema de seleção adversa (Arrow, 1985; Jensen \& Meckling, 1976).

Assim, a teoria de agência pode ser utilizada conjuntamente com a abordagem contingencial, dado que a relação contratual principal-agente pode ser estudada não apenas focando esse tipo específico de relação, mas também a contextualizando, ao considerar o ambiente no qual ela ocorre, como na gestão pública.

Ademais, os contratos envolvidos pela teoria de agência, assim como na gestão pública e no caso do acordo de resultados, são permeados por vários problemas como racionalidade limitada, comportamento oportunista, risco moral e seleção adversa.

A racionalidade limitada dos agentes pode ser representada pelo fato de estes não conseguirem prever, ex ante, todas as circunstâncias ou ações que ocorrem em uma relação, não permitindo que uma 
escolha racional seja efetuada, pois faltam informações completas e habilidades aos indivíduos (Simon, 1955). Para Williamson (1985), o comportamento oportunista dos agentes pode ser representado pela ação intencional daqueles que buscam seus próprios interesses e agem em benefício próprio nas relações, usufruindo de possíveis falhas contratuais. $\mathrm{O}$ risco moral está relacionado às ações praticadas pelos agentes quando estas não são de conhecimento do principal ou estão ligadas ao oportunismo póscontratual dos agentes, representado pelas mudanças de comportamentos após o estabelecimento de determinado contrato em que não podem ser monitoradas (Arrow, 1985). A seleção adversa refere-se às informações que os agentes detêm sobre suas características individuais, as quais permitem agir de maneira oportunista no estabelecimento do contrato (Arrow, 1985).

Ayee (2005) acrescenta que ambiguidade, comportamento oportunista, risco moral e seleção adversa também permeiam a gestão pública, na qual as relações de agência podem se caracterizar de maneiras distintas. Uma possibilidade é o modelo no qual a sociedade assume o papel de principal e o gestor público representa o agente, uma vez que recebe da população autorização para agir em seu nome, sendo utilizados meios para prestar contas acerca das decisões tomadas (Ayee, 2005; Przeworski, 1996). Outro formato possível ocorre quando o político, enquanto representante do Estado, representa o principal, e os agentes atuantes no mercado (empresários) atuam como agentes propriamente ditos, sendo requeridos instrumentos de regulação para gestão dessa relação (Przeworski, 1996). Outro modelo possível ocorre quando o representante da sociedade - neste caso, o político - assume a figura de principal e os gestores públicos contratados pelo principal para executar as tarefas envolvidas na gestão pública representam os agentes (Ayee, 2005; Przeworski, 1996).

No caso específico do Acordo de Resultados, estabeleceu-se uma série de estratégias, metas e indicadores para avaliar, do alcance delas, além de um sistema de incentivos financeiros como premiação. Porém, é possível que os agentes ou gestores públicos tentem influenciar tanto a elaboração quanto o alcance das metas pactuadas para facilitar o recebimento da bonificação. Desse modo, os agentes agiriam de maneira oportunista, conforme prevê a teoria de agência. Todavia, é possível que existam problemas na estruturação das metas e indicadores pactuados, o que levaria a alterações deles em razão de fatores internos ou externos à organização.

Assim, a teoria da contingência e a teoria de agência representam um importante arcabouço teórico a ser utilizado para verificar os fatores que influenciam as alterações das metas e dos indicadores utilizados na avaliação de desempenho inseridos no Choque de Gestão.

\section{Metodologia}

Considerando os objetivos propostos, esta pesquisa pode ser classificada como explicativa, uma vez que busca identificar possíveis fatores que contribuem para a ocorrência de determinado fenômeno - neste caso, para a alteração das metas e dos indicadores utilizados para avaliação de desempenho da organização estudada (Gil, 2002).

Quanto aos procedimentos técnicos, trata-se de uma abordagem que se direciona para o estudo de caso, técnica que busca alcançar profundidade na análise.

Apesar de não permitir a elaboração de uma contribuição generalizável, o estudo de caso permite a compreensão e o aprofundamento no caso de mudanças na contabilidade gerencial de determinada organização (Scapens \& Roberts, 1993). Neste trabalho, buscam-se especificamente a compreensão e o aprofundamento das alterações ocorridas nos indicadores utilizados na avaliação de desempenho, o que justifica a utilização do método.

Inicialmente, foram escolhidas as secretarias-alvo para a realização das entrevistas. Para a seleção das secretarias, foram considerados os seguintes critérios: (a) apresentavam maiores níveis de crédito autorizado para as despesas a serem executadas; (b) abrangem diferentes partes do processo de gestão governamental; e (c) mantêm contato mais próximo com a sociedade. Assim, foram realizadas 
entrevistas nas seguintes secretarias: Secretaria de Estado de Defesa Social (Seds), Secretaria de Estado de Educação (SEE), Secretaria de Estado de Fazenda (SEF), Secretaria de Estado de Saúde (SES), e Secretaria de Transporte e Obras Públicas (Setop). Também foram realizadas entrevistas na Secretaria de Estado de Planejamento e Gestão (Seplag), pelo fato da mesma ser a responsável pela gestão do funcionamento do Acordo de Resultados.

Complementarmente, a escolha dessas secretarias seguiu a recomendação de um grupo de especialistas integrado por professores-pesquisadores da área da Gestão Pública, familiarizados com os indicadores de desempenho, os quais consideraram essas secretarias como componentes de áreas prioritárias, voltadas para a execução das estratégias estabelecidas. Em 2013, Minas Gerais trabalhava com o modelo denominado Estado em Rede de Desenvolvimento Integrado, o qual integrava os objetivos e as estratégias do planejamento do Estado em áreas de resultados, as quais contêm metas e o desdobramento delas em objetivos, estratégias e indicadores de desempenho para cada uma (Secretaria de Estado de Planejamento e Gestão, 2011)

Grande parte dos objetivos estratégicos mencionados estava relacionada às secretarias analisadas, uma vez que os esforços e os recursos para o alcance das estratégias estabelecidas encontravam-se distribuídos em onze áreas de resultados: (a) Rede de Educação e Desenvolvimento Humano; (b) Rede de Atenção em Saúde; (c) Rede de Defesa e Segurança; (d) Rede de Desenvolvimento Social e Proteção; (e) Rede de Desenvolvimento Econômico Sustentável; (f) Rede de Ciência, Tecnologia e Inovação; (g) Rede de Desenvolvimento Rural; (h) Rede de Identidade Mineira; (i) Rede de Cidades; (j) Rede de Infraestrutura; e (k) Rede de Governo Integrado, Eficiente e Eficaz (Secretaria de Estado de Planejamento e Gestão, 2011).

Para a realização da pesquisa, optou-se pela entrevista semiestruturada, por ser definida como um método que contém questionamentos básicos apoiados em teorias e hipóteses que se relacionam ao tema da pesquisa (Triviños, 1987), além de possibilitar a obtenção de respostas contendo informações de forma mais livre, sendo que estas não estão condicionadas a uma padronização de alternativas (Manzini, 1990).

Para execução das entrevistas, utilizou-se um roteiro de entrevista semiestruturado, contendo 27 questões distribuídas em dois grandes blocos: o primeiro com questões relacionadas à identificação do respondente, como nome, cargo, tempo de função e secretaria à qual pertencia; e o segundo com aspectos ligados à construção das metas e indicadores utilizados na avaliação de desempenho, além de questões relacionadas aos possíveis fatores que pudessem influenciar as alterações dos indicadores ao longo do tempo.

As questões referentes aos possíveis fatores que pudessem levar à alteração dos indicadores utilizados na avaliação de desempenho foram construídas de acordo com a estrutura teórica utilizada e, ainda, as variáveis já identificadas na literatura, capazes de influenciar as alterações dos indicadores.

Adicionalmente, optou-se pela elaboração de um pré-teste, para verificar a validade do roteiro de entrevistas. Duas entrevistas-teste foram realizadas para a execução de eventuais ajustes no roteiro de entrevista. Posteriormente, procedeu-se ao andamento dos agendamentos e das entrevistas com os servidores contidos na amostra.

Foram entrevistados 21 servidores públicos, distribuídos nas seis secretarias analisadas. Em um primeiro momento, buscou-se entrevistar no mínimo três servidores de cada secretaria da amostra, notadamente os assessores à frente das Assessorias de Gestão Estratégica, e mais dois servidores posicionados acima do nível de diretoria. Isso pelo fato de estes serem os níveis hierárquicos envolvidos nas definições dos objetivos, indicadores e metas contidos no Acordo de Resultado. Em algumas secretarias, mais de três servidores se mostraram dispostos a ser entrevistados, fazendo com que mais servidores fossem entrevistados, como na Secretaria de Estado de Educação, na Secretaria de Estado de Fazenda e na Secretaria de Estado de Transporte e Obras Públicas. 
Para a escolha dos servidores a serem entrevistados, realizou-se um levantamento dos organogramas das secretarias, disponíveis em seus endereços eletrônicos, sendo coletados os seguintes dados: nome do servidor, posição hierárquica, função, telefone e $e$-mail.

Em um primeiro momento, foram realizados contatos telefônicos com os servidores para convidálos a participar das entrevistas e confirmar a data e o horário. Para tanto, focou-se no contato com os assessores das Assessorias de Gestão Estratégica e Inovação (Agei) de cada uma das secretarias componentes da amostra, pelo fato de eles serem considerados os gestores do Acordo de Resultados em suas respectivas secretarias. Alternativamente, foram realizados os demais contatos.

Definidos os níveis hierárquicos escolhidos para compor a amostra, foram entrevistados aqueles que aceitaram o convite para participar da pesquisa. Porém, houve a participação dos assessores das Ageis de todas as secretarias da amostra. Excepcionalmente, em três secretarias específicas foram entrevistados servidores que não estavam alocados hierarquicamente acima do nível de diretoria. Isso ocorreu porque os servidores alocados hierarquicamente acima do nível de diretoria contatados demonstraram indisponibilidade em participar da pesquisa. Nesses casos específicos, a escolha dos servidores a serem entrevistados ocorreu com base em indicações apresentadas pelos assessores das Ageis, por considerarem que eles possuíam conhecimento e grau de participação no Choque de Gestão e no Acordo de Resultados suficientes para colaborarem com a pesquisa.

Na Tabela 3, são apresentados os níveis hierárquicos dos servidores entrevistados e a duração das entrevistas realizadas.

Tabela 3

Níveis Hierárquicos dos Servidores Entrevistados e Duração das Entrevistas

\begin{tabular}{lccc}
\hline $\mathbf{N}$ & Entrevistado & Nível Hierárquico & Duração (minutos) \\
\hline 1 & Entrevistado 1 & Subsecretário & 53 \\
2 & Entrevistado 2 & Subsecretário & 43 \\
3 & Entrevistado 3 & Assessor & 37 \\
4 & Entrevistado 4 & Diretor & 29 \\
5 & Entrevistado 5 & Assessor & 13 \\
6 & Entrevistado 6 & Superintendente & 45 \\
7 & Entrevistado 7 & Assessor & 28 \\
8 & Entrevistado 8 & Outros & 40 \\
9 & Entrevistado 9 & Assessor & 17 \\
10 & Entrevistado10 & Assessor & 19 \\
11 & Entrevistado 11 & Assessor & 34 \\
12 & Entrevistado 12 & Assessor & 37 \\
13 & Entrevistado 13 & Assessor & 17 \\
14 & Entrevistado14 & Outros & 36 \\
15 & Entrevistado 15 & Outros & 27 \\
16 & Entrevistado 16 & Assessor & 42 \\
17 & Entrevistado 17 & Assessor & 24 \\
18 & Entrevistado 18 & Superintendente & 17 \\
19 & Entrevistado 19 & Superintendente & 26 \\
20 & Entrevistado 20 & Diretor & 28 \\
21 & Entrevistado 21 & Subsecretário & 40 \\
\hline & Total & & $\mathbf{6 5 2}$ \\
\hline
\end{tabular}

Nota. Elaborada pelos autores.

RAC, Rio de Janeiro, v. 21, Edição Especial FCG, art. 1, pp. 1-21, Abril $2017 \quad$ www.anpad.org.br/rac $($ (c) Er 
Para garantir a não identificação, os entrevistados foram classificados em ordem alfabética e, posteriormente, enumerados. As entrevistas ocorreram nos meses de novembro e dezembro do ano de 2013. Com relação à sequência das entrevistas, estas ocorreram de acordo com a disponibilidade dos entrevistados. As entrevistas foram realizadas na sede da estrutura administrativa do Estado, sendo todas gravadas com a autorização dos entrevistados. É importante destacar que foi acordado com todos eles a garantia de anonimato, não podendo ser expostos quaisquer dados contendo características, informações ou detalhes que pudessem levar à caracterização dos respondentes ou das secretarias às quais pertenciam. As entrevistas foram transcritas para a realização das análises previstas, em que se utilizou a técnica de análise de conteúdo. Esta busca ultrapassar o nível estabelecido pelo senso comum e o subjetivismo nas interpretações, para que sejam extraídos elementos de uma análise científica em documentos, textos literários, biografias, entrevistas ou observações, podendo ser utilizada com enfoque qualitativo, no qual é considerada a presença de determinada característica ou conteúdo na comunicação analisada (Bardin, 1977).

Ainda com relação aos procedimentos, foi utilizada a análise de conteúdo, baseada no método de análise temática ou categorial, o qual consiste no desdobramento do texto em unidades representadas pelas categorias, e permite a classificação das unidades de codificação em categoriais de elementos de significação (Bardin, 1977).

Desse modo, utilizou-se a técnica de análise de conteúdo, baseada no método categorial, para a identificação da percepção dos entrevistados quanto aos fatores que podem influenciar a construção e as alterações dos indicadores utilizados na avaliação de desempenho ao longo do tempo e analisar as evidências de regularidades ou padrões e a identificação e análise das possíveis inconsistências nas falas dos entrevistados.

A operacionalização das categorias de análise foi realizada considerando a premissa apresentada no referencial teórico de que os gestores podem realizar alterações ou ajustes em ferramentas utilizadas, como os indicadores e as metas, na avaliação de desempenho, por fatores ambientais e organizacionais.

Segundo o arcabouço teórico da teoria da contingência, os processos de mudanças da organização são influenciados por fatores ambientais e organizacionais que afetam a relação entre as características da organização e o desempenho (Donaldson, 2001; Wright et al., 1998/2009). Esses fatores podem determinar se as organizações adotam ou não mudanças em suas estruturas.

Este estudo se baseia nos seguintes pressupostos existentes no arcabouço da teoria da contingência, quais sejam: (a) há associação entre fatores contingentes e práticas gerenciais; (b) os fatores contingentes definem o sistema ou a prática gerencial adotada; e (c) há existência de ajuste ou adequação de algum nível do sistema ou da prática gerencial adotada ao nível de contingência que pode conduzir ao aumento do desempenho, ou existe desajuste que pode conduzir à queda do desempenho.

No caso específico do Acordo de Resultados, elemento contido no Choque de Gestão, realizado na estrutura gerencial do Estado de Minas Gerais, seria esperado que os agentes promovessem mudanças na estrutura interna, como nos indicadores utilizados na avaliação de desempenho, em função de diversos fatores presentes nos contextos interno e externo da entidade.

Para a identificação da percepção dos gestores quanto à influência ou não daquele fator ou variável sobre o processo de construção e alterações dos indicadores, foi realizada a categorização dos trechos presentes nas entrevistas, considerando as variáveis descritas na literatura. O processo de categorização das entrevistas foi realizado com o auxílio do software QDA Miner (versão para teste), utilizado na análise de dados qualitativos que possibilita a codificação, a anotação e a análise de documentos e imagens. 


\title{
Análise dos Resultados
}

Durante as entrevistas, os respondentes foram questionados quanto à ocorrência de alterações dos indicadores utilizados na avaliação de desempenho nas secretarias analisadas. Segundo a maioria dos respondentes, ocorrem alterações nos indicadores utilizados, conforme destacado a seguir:

\begin{abstract}
"Surgem novos indicadores. Você vai ver que de um ano para o outro, às vezes, tem um novo, outros são excluídos, e as metas às vezes são elevadas com o valor de referência. São essas, basicamente, as mudanças que ocorrem.... Pode acontecer. Você pode excluir um indicador. Tem esta possibilidade de excluir o indicador. Depende da justificativa. É possível alterar tudo, mas tem instâncias que vão aprovar isso" (Entrevistado 9).

“É um exemplo esse indicador que eu comentei de ..., aqui da secretaria .... É, teve uma redução da meta e também uma alteração da fórmula do indicador. Então, a redução da meta, se não me engano, foi de 20 pra 15 , e a fórmula do indicador foi alterada, porque antes não considerava os dias paralisados e agora passou a considerar" (Entrevistado 15).

"Tem um procedimento com burocracia enorme pra (sic) trocar uma meta. Nisso eles são muito cuidadosos. Tem um formulário de solicitação de mudanças que você justifica por que aquela meta deixou de ser razoável. [E], ao mesmo tempo que eu trabalhava com Acordo de Resultados, como que eu avaliava uma solicitação de mudança que chegava pra (sic) mim? Eu avaliava se a mudança de cenário era passível de previsão no momento em que aquilo foi pactuado, porque se não, se fosse, era mal planejamento do indicador ou se tratou de uma superveniência ou uma insubsistência de alguma coisa que não era passível de previsão que realmente prejudicou aquela possibilidade de chegar naquele resultado. Então, a gente revê a pactuação pra (sic) que continue desafiador, sem frustrar o servidor de achar que aquilo virou impossível" (Entrevistado 7).
\end{abstract}

Nota-se, pela fala do Entrevistado 7, que os indicadores devem ser factíveis e passíveis de reconhecimento por parte dos avaliados. Ou seja, há evidências de uma preocupação com as características dos indicadores, conforme discutido por Jannuzzi $(2002,2005)$. Outra questão destacada pela maioria dos entrevistados é que podem ocorrer alterações nos indicadores utilizados na avaliação de desempenho, bem como das metas estipuladas, conforme destacado por autores como Jackson e Lapsley (2003), Bjørnenak (1997) e Malmi (1999). Porém, para o Entrevistado 7, existe um processo de avaliação nesse procedimento, sendo possível que ocorram alterações somente nos casos em que existe influência de elementos não previsíveis pelos gestores.

Segundo a maioria dos entrevistados, o processo para promover uma alteração envolve alguns procedimentos e um longo período de discussão, já comentado pelo Entrevistado 7 e acrescentado por outros entrevistados:

"Tem um sistema de gestão estratégica. A gente cadastra essa mudança. Esse é o procedimento que segue pra (sic) Seplag avaliar se é pertinente ou não" (Entrevistado 9).

"As alterações ocorrem de duas formas, a cada revisão do Acordo. Então, anualmente, a gente tem um momento de revisão e a gente faz essas alterações. Mais ao longo do monitoramento, no período da avaliação, é possível ocorrerem solicitações, muito em função de um planejamento de risco, que a gente faz minimamente ... Isso vai causar um desempenho menor, e há um risco superveniente à vantagem do órgão ao qual não teria ação, mas existe um processo que essa alteração de meta tem que ser validada" (Entrevistado 14).

"Sempre para exercícios seguintes. No exercício, o órgão pode entrar com recurso durante o período de avaliação do Acordo falando por que o indicador não é o mais adequado e que poderia ter sido medido de uma outra forma. Esse recurso pode ser aceito ou não.... Tem sido aceito nos últimos anos. Tem dez anos que a gente pactua. Então, já tem um grande aprendizado nisso. Então, ele pode entrar com um recurso falando quais foram as causas exógenas, a ação dela, que fizeram com que eles perderam (sic) a meta, que provavelmente trocaram o indicador. Ele pode argumentar: 'Caiu uma ponte'; 'Isso não estava no escopo'; 'Teve uma externalidade que eu não controlo'; 'Houve um desastre ambiental, então eu não consegui 
terminar a obra'. Então ele pode entrar com coisas nesse sentido, e a comissão vai avaliar se isso é pertinente ou não" (Entrevistado 2).

O processo de alteração envolve negociação. Dependendo da justificativa apresentada, o órgão regulador do Acordo de Resultados (a Seplag) autoriza ou não a alteração. Porém, a autorização para a alteração só ocorre mediante justificativa que explique claramente o motivo da alteração.

Apesar de a maioria dos entrevistados indicar a não possibilidade de alterações durante o processo, há uma evidência no depoimento do Entrevistado 14 apontando para a possibilidade de ocorrência de tais alterações em alguns casos específicos, bem como nos de outros entrevistados, conforme trechos destacados anteriormente. É possível que as particularidades de algumas secretarias possibilitem essas alterações. Segundo o arcabouço teórico da teoria da contingência, existem fatores contextuais ou comportamentais, como características do sistema administrativo-político, incerteza ambiental e estrutura tecnológica, que podem levar às alterações de mudanças de práticas, como as alterações dos indicadores utilizados na avaliação de desempenho (Christensen \& Yoshimi, 2003; Lüder, 1992; Upping \& Oliver, 2011).

Um dos problemas para os avaliados verificados nesse processo é que, durante um mesmo Acordo ou exercício, caso se verifique que determinado indicador não pode ser alcançado, a negociação para a revisão da meta ou a alteração do indicador só ocorre no final do período, quando o indicador já não pode mais ser alcançado, conforme afirmado por um dos entrevistados:

“A gente conversa com o nosso subsecretário. Ele autoriza, a gente passa para a Agei, e a Agei conversa com a Seplag. Mas esse é um problema que tem em Minas Gerais. A gente só conversa depois que acabou o período. Eles não trocam durante" (Entrevistado 19).

Quando questionados sobre os principais fatores que influenciaram as alterações ocorridas nos indicadores, vários fatores foram citados.

Para um entrevistado, com o decorrer do tempo as metas podem ficar muito fáceis de ser alcançadas, sendo necessário ajustá-las (ou os indicadores) às novas capacidades de trabalho, ou ajustar os próprios indicadores a partir da experiência obtida com sua aplicação anterior, conforme descrito pelo entrevistado mencionado a seguir:

\footnotetext{
“A gente teve uma mudança na própria metodologia por parte do governo para prever a própria mudança estratégica. Então, foi um ano que a gente criou vários indicadores que não vinham sendo acompanhados nos anos anteriores, 2010, 2009 [e] 2008. Quando a gente criou, tínhamos uma expectativa, mas não mensuravam desempenho. Então, aqueles que eram considerados como fracos, onde a meta era batida com muita facilidade, a gente vai depurando o Acordo pra (sic) excluir esse tipo de indicador e deixar apenas os que tragam desafio e ganho de eficiência e atividade da equipe. As metas dos indicadores a gente também muda (sic), sempre no sentido de melhoria. São muitos poucos casos em que a gente traz uma piora no indicador, e esses casos, $100 \%$ das vezes, é uma redução no volume de investimento por parte do governo, que tem uma série de prioridades. Então, existe, às vezes, uma redefinição das prioridades, e isso traduz uma redução de investimento em determinado setor. Então, você sente um desempenho melhor com investimento menor que [em] alguns casos. É possível. Então, pra (sic) acompanhar isso, a gente reduz a meta. Então, 5\%, 10\% das alterações que a gente faz, a maior parte é de apertar mais a meta, tornar mais difícil, para que o desempenho seja melhor" (Entrevistado 14).
}

Percebe-se que, em alguns casos, é possível alterar a meta sem mudar o indicador e que, em outros, se a meta é facilmente alcançada, o indicador pode ser entendido como irrelevante e retirado do modelo. Segundo o Entrevistado 14, essas alterações ocorrem para tornar a meta mais difícil de ser alcançada, o que a gestão do Acordo de Resultados entende como ação para obter a melhoria do desempenho.

Para um grupo específico de entrevistados, as alterações dos indicadores fazem parte do amadurecimento do modelo, conforme afirmado:

“Algumas finalizaram. Então, você tem que oferecer metas e indicadores novos. E outras foi porque foram consideradas inatingíveis" (Entrevistado 4). 


\begin{abstract}
“Alteração de meta, o objetivo acho que é sempre colocar uma coisa mais desafiadora para a equipe. E alteração da fórmula ou da descrição do indicador, eu acho que é pra tentar fazê-lo mais eficiente mesmo. Porque acho que durante esses anos, desde o Choque de Gestão aqui no Estado ... durante esse processo de amadurecimento, é normal você ir aprimorando cada um desses indicadores" (Entrevistado 15).

"Elas ocorrem em decorrência da própria avaliação do Acordo. Quando você vai ler o Acordo, você percebe que acompanhar aquele indicador, aquela meta, não se mostrou como a melhor forma de acompanhar aquela política. Tem-se a proposta de fazer alteração, tanto o regulado como o regulador. A partir do processo de avaliação, saem algumas diretrizes. Isso não se mostrou produtivo, ou porque é do processo natural de avaliação, como é no caso da secretaria" (Entrevistado 2).
\end{abstract}

Segundo alguns entrevistados, as alterações dos indicadores utilizados na avaliação de desempenho podem ocorrer para facilitar o alcance das metas estipuladas ou facilitar o recebimento do incentivo atrelado ao alcance das metas (exemplificando o que foi discutido em parte da literatura como em Christensen |\& Yoshimi, 2003; Lüder, 1992; Naranjo-Gil, 2009), denotando oportunismo, conforme afirmado:

"Ele passou a ser assim: se a secretaria não atingir 100\% dos indicadores finalísticos, nada de prêmio. A gente tinha mais de vinte indicadores finalísticos. Quer dizer, a probabilidade de ter algum que a gente não ia alcançar era gigantesca. Então, foi uma decisão bem pragmática mesmo. Pra (sic) gente conseguir continuar recebendo o prêmio, a gente teve que limitar, e acho que no fim das contas isso foi excelente. $\mathrm{O}$ Acordo ... ele ficou mais digerível porque ficou muito mais simples de compreender" (Entrevistado 6).

De acordo com o Entrevistado 6, a redução do número de indicadores utilizados na avaliação desempenho foi, de certa maneira, salutar para o processo. De um lado, ele ressalta que essa redução aumentou a probabilidade de alcançar as metas pactuadas no Acordo de Resultados; de outro, argumenta que a adoção de um número menor de indicadores melhorou o Acordo de Resultados, pois passou a haver maior foco nos objetivos envolvidos nos indicadores.

Outro entrevistado relata a possibilidade da existência de oportunismo por parte dos agentes públicos no processo de alteração dos indicadores:

“Acabei de pensar agora. Por exemplo o indicador ... é indicador das secretarias ... e da.... Esse, sim, todo mundo fica de olho. Mas aí a meta já é dada pela lei. Então, ela acaba não mudando, porque, às vezes, você pode observar: é mudar a fórmula para que a meta se adeque.... Mas isso não é muito claro, porque você nunca vai achar alguém dizer que mexeu na fórmula de calcular o indicador.... Essa especificamente não; ela é uma contabilidade criativa. Agora, detalhe, ser criativo, mas validada pelo ministério" (Entrevistado 16).

É possível perceber, pela fala do Entrevistado 16, problemas relacionados ao oportunismo dos agentes envolvidos no Acordo, sendo que existem casos específicos em que a meta é estabelecida pela legislação, e os indicadores podem ser alterados para adequá-los a ela. Nesses casos, mesmo que os agentes ajam de maneira legal para adequar determinado indicador a uma meta que não pode ser alterada pela rigidez legal, o Acordo poderia ser influenciado negativamente para beneficiar os interesses dos agentes (Entrevistados 6 e 16).

Segundo a estrutura conceitual da teoria de agência, uma parte da relação (o principal) delega autoridade à outra (os agentes) para que estes possam desempenhar as atividades requeridas a seu favor (Jensen \& Meckling, 1976). Considerando especificamente a gestão pública, os agentes (gestores públicos) podem desempenhar ações distintas daquelas que seriam escolhidas pelo principal, utilizando as informações que possuem em favor de seus próprios interesses, ou seja, agindo de maneira oportunista, porém utilizando-se de ações previstas pela legislação, conforme ressaltado pelo Entrevistado 16 (Ayee, 2005).

Para um dos entrevistados, as alterações dos indicadores podem ocorrer por erro na estruturação do indicador ou pelo fato de ele não refletir a realidade, conforme destacado: 
"Dos indicadores? Uai! Pode ser tanto de uma ordem superior. Veem que não é isso mais que a gente quer... pra lá, né (sic)? Quanto a gente perceber que o indicador não reflete exatamente a meta nem o processo que a gente quer" (Entrevistado 19).

Ademais, alguns dos entrevistados afirmaram que as alterações dos indicadores podem ocorrer em função de mudança de contexto, ao qual está alocado o indicador:

"Essas alterações ocorrem, muitas vezes, porque é um processo de maturação. Então, coloca uma meta e vai ao longo do tempo identificando que ela tem que ser mudada, e o mapa serve até pra (sic) refletir não só os problemas, mas os gargalos também. Vou te dar um exemplo, como que (sic) mudou o contexto: Eu tinha uma meta de recursos humanos que era a quantidade de cursos providos pela secretaria dos servidores. A meta era $\mathrm{x}$, e a gente identificou em determinado ano que estava sendo feito 10x. Então, o que aconteceu? Eu comecei a ter uma tecnologia de educação a distância. Mudou toda lógica. Isso mudando, tenho que mudar toda a meta. Não são só alteração de indicador, mas mudança de contexto.... Não adianta nada eu manter aquela meta ali. Então, eu tenho mudanças não só de amadurecimento do indicador, mas de efeitos exógenos aî" (Entrevistado 21).

"O indicador muda pela mesma razão da meta: superveniência e subsistência de contexto. Fator exógeno é basicamente isso, tanto indicador quanto meta" (Entrevistado 7).

O Entrevistado 7 afirmou que as alterações podem ocorrer, em parte, pela mudança de contexto ou pelo surgimento de fatores exógenos, corroborando os achados de Upping e Oliver (2011), que indicam que as mudanças na estrutura contábil de entidades públicas podem ocorrer em função de fatores e pressões externas, ou exógenas, e internas. Contudo, segundo o entrevistado, em alguns casos há má gestão no processo de construção e acompanhamento dos indicadores. E, ainda, existe influência da percepção do agente que constrói o indicador, conforme descrito a seguir:

"Eu acho que a gestão de conhecimento no Estado é muito ruim. Então, o processo de construção daquele indicador, porque ele foi descrito daquela forma, porque aquilo foi entendido por aquela equipe no momento que pariu aquele indicador, que aquela era a melhor forma de descrevê-lo, que era a melhor fórmula e etc. Aquilo não é registrado: só registra o indicador. Então, provavelmente no próximo ano quem vai olhar aquilo vai ser outra pessoa. Tem uma rotatividade alta nas outras equipes. Então, essa outra pessoa diz: "Faltou isso mesmo". Se você parar para pensar, não é desse universo. Se você considera esse universo na fórmula, você vai ter uma distorção x. A gente tem que mudar essa fórmula. Eu acho que isso que faz os indicadores mudarem" (Entrevistado 7).

Após as análises dos extratos das entrevistas apresentadas, é possível perceber que as alterações dos indicadores utilizados na avaliação de desempenho podem ocorrer por diversos motivos, por exemplo, aperfeiçoamento do modelo ou do indicador, ocorrência de fatores exógenos, facilitação do atingimento das metas e do recebimento de incentivos.

De acordo com as teorias que embasam esta pesquisa - teoria da contingência e teoria de agência, esses fatores podem ser considerados como motivadores das mudanças ou alterações.

Segundo o arcabouço teórico da teoria da contingência, fatores internos e externos presentes no contexto organizacional podem ser considerados como influentes em processos de mudanças de práticas gerenciais, assim como encontrado nas entrevistas realizadas (Christensen \& Yoshimi, 2003; Lüder, 1992; Upping \& Oliver, 2011). Ainda, é previsto pela teoria de agência que ações que envolvam comportamento oportunista dos agentes, como adequação dos indicadores às metas, a fim de garantir o alcance delas e o recebimento dos incentivos, estão presentes na gestão pública e podem influenciar as alterações das práticas utilizadas (Ayee, 2005; Lüder, 1992). Na Tabela 3, apresentada a seguir, são descritos os fatores que os entrevistados consideram que influenciam as alterações dos indicadores, bem como a classificação deles, de acordo com as teorias utilizadas: 
Tabela 4

Fatores que Influenciam as Alterações dos Indicadores de Desempenho Citados

\begin{tabular}{lll}
\hline Fatores Citados & Entrevistados & Indícios Teóricos \\
\hline As metas ficam fáceis de serem alcançadas. & 14 & $\begin{array}{l}\text { Não identificado nas bases } \\
\text { teóricas da pesquisa. }\end{array}$ \\
\hline $\begin{array}{l}\text { Aperfeiçoamento do modelo ou erros identificados na } \\
\text { estrutura do indicador ou o mesmo não reflete a realidade. }\end{array}$ & $1,2,4,5,15$ e 19 & $\begin{array}{l}\text { Não identificado nas bases } \\
\text { teóricas da pesquisa. }\end{array}$ \\
\hline Facilitar o alcance da meta ou o recebimento dos incentivos. & 6 e 12 & Teoria de agência. \\
\hline Adequação dos indicadores às metas. & 16 & Teoria de agência. \\
\hline Fatores exógenos e mudanças de contexto. & 7 e 21 & Teoria de contingência. \\
\hline Má gestão no processo de construção do indicador. & 7 & $\begin{array}{l}\text { Não identificado nas bases } \\
\text { teóricas da pesquisa. }\end{array}$ \\
\hline
\end{tabular}

Nota. Elaborada pelos autores.

É possível perceber que alguns dos fatores mencionados pelos entrevistados são destacados pela estrutura teórica utilizada enquanto fatores que podem influenciar as alterações dos indicadores utilizados na avaliação de desempenho.

\section{Conclusões}

Ao avaliar o modelo gerencial utilizado no governo de Minas Gerais, considerando sua condução e seus resultados alcançados, é possível constatar que, apesar de os indicadores de desempenho fundamentais para a gestão de qualquer organização - terem sido previamente definidos de forma alinhada aos interesses do Estado, eles sofrem ajuste durante a gestão para atender aos objetivos dissociados daqueles do Estado.

De acordo com a revisão teórica acerca da teoria da agência e da teoria da contingência e tomando como base os elementos empíricos aportados ao longo do desenvolvimento deste trabalho, foi possível identificar evidências de que várias pressões e interesses divergentes aos interesses do Estado podem motivar tais ajustes, que podem comprometer os objetivos iniciais pautados no Acordo de Resultados.

A despeito do esforço do governo do Estado de Minas Gerais de modernizar a gestão das secretarias, houve a criação e a valorização de indicadores para avaliação de desempenho. Entretanto, a maneira como foram alterados, em alguns casos ao longo do tempo, fez com que os indicadores se mostrassem voláteis e não propiciassem o benefício esperado como instrumento de controle e como promotor de melhorias no processo de gestão. Nesse contexto, tal esforço não foi capaz de blindar a estrutura gerencial do modelo de gestão diante de algumas pressões identificadas pelas bases da teoria da agência e da teoria da contingência, desvirtuando o modelo inicialmente proposto e promovendo alterações nos indicadores.

Como resultado, um dos efeitos gerados se refere ao comprometimento da accountability, ou prestação de contas, que deveria ser gerada ao longo da gestão do Estado. Nesse caso, se os resultados da avaliação do alcance dos objetivos e das metas fossem definidos de maneira segura, maior transparência e relações de controle mais consistentes dentro da estrutura gerencial do Estado poderiam ser alcançadas. Assim, se o acompanhamento do Acordo ocorresse de maneira mais efetiva e transparente, a possibilidade de alterações das metas e dos indicadores seria reduzida, o que poderia aumentar o desempenho geral do modelo. 
Se a accountability estiver comprometida pela fragilidade da estrutura gerencial por meio das alterações dos indicadores, ela também pode atuar como parte de um conjunto de possíveis soluções para mitigar os problemas detectados ao longo das análises realizadas, agindo como um mecanismo de proteção contra alterações indevidas nos indicadores de desempenho.

Considerando as implicações de ordem gerencial ou técnica, apesar de os entrevistados concordarem que o Acordo de Resultados é um modelo de gestão que melhorou, de forma geral, a execução das ações, o que levou à melhoria do desempenho da gestão, nota-se que sua operacionalização e sua gestão, no que se refere aos indicadores e metas, contêm algumas deficiências que precisam ser consideradas. Cita-se, o fato de não se permitir aos agentes envolvidos no Acordo a possibilidade de reduzir os níveis das metas pactuadas, em comparação com períodos anteriores. Dado que não é possível reduzir os níveis pactuados, os gestores públicos podem imputar folga nas metas propostas se entenderem que o alcance dos níveis pactuados parece fácil, podendo, com isso, gerar elevações dos níveis para os próximos períodos. Nesse caso, se for possível reduzir as metas e os indicadores pactuados, é possível que os níveis a serem pactuados se tornem mais próximos da realidade.

Concluindo, pode-se observar a ocorrência de problemas em três dimensões do modelo. A primeira se refere à falta de integração entre o horizonte temporal dos objetivos estratégicos, definidos para o longo prazo, e as metas e os indicadores, definidos para períodos distintos e voltados para o curto prazo. A segunda prende-se à forma como são construídos os elementos que o compõe, desconsiderando o alinhamento entre estes e as particularidades de cada uma das áreas/secretarias. A terceira tem a ver com a forma como é realizado o acompanhamento da execução das atividades previstas e da avaliação final. Esses problemas abrem espaço para que as metas e os indicadores sejam alterados e descontinuados ao longo do tempo, ora por comportamento oportunista do gestor, ora para corrigir falhas e amenizar as possíveis consequências dessas disfuncionalidades.

Espera-se que este estudo contribua com a literatura sobre avaliação de desempenho no setor público. Contudo, as análises precisam ser consideradas no contexto em que foram realizadas. Ainda, este estudo possui uma série de limitações que necessitam ser consideradas. Citam-se a seleção das secretarias, baseando-se em requisitos que podem ter desconsiderado outros aspectos que podem ser relevantes, como o período de aplicação da avaliação de desempenho nas secretarias.

Para a realização de futuras pesquisas, sugere-se analisar se as secretarias que executam ações e entregam serviços mais próximos da sociedade, que poderiam influenciar a percepção quanto à influência da pressão social, promoveriam mais alterações dos indicadores e metas no decorrer do tempo. Por fim, indica-se o grau de controlabilidade dos indicadores utilizados. Futuras pesquisas poderiam também verificar a influência do grau de controlabilidade dos indicadores de desempenho e da utilidade econômica percebida pelos agentes sobre as alterações dos indicadores e metas, bem como se há diferenças na influência sobre as alterações dos indicadores.

\section{Referências}

Arrow, K. J. (1985). The economics of agency. In J. W. Pratt \& R. J. Zeckhauser (Eds.), Principals and agents: the structure of business (Chap. 2, pp. 37-51). Boston, MA: Harvard Business School Press.

Astley, W. G., \& Van de Ven, A. H. (2005). Debates e perspectivas centrais na teoria das organizações. Revista de Administração de Empresas, 45(2), 52-73.

Ayee, J. R. A. (2005). Public sector management in Africa [Working Paper $n^{\circ}$ 82]. Economic Research Working Paper, African Development Bank, Tunisia.

Bardin, L. (1977). Análise de conteúdo. Lisboa: Editora Edições 70. 
Barzelay, M. (1997). Central audit institutions and performance auditing: a comparative analysis of organizational strategies in the OECD. Governance, 10(3), 235-260. http://dx.doi.org/10.1111/0952-1895.411997041

Bjørnenak, T. (1997). Diffusion and accounting: the case of ABC in Norway. Management Accounting Research, 8(1), 3-17. http://dx.doi.org/10.1006/mare.1996.0031

Boland, T., \& Fowler, A. (2000). A systems perspective of performance management in public sector organisations. International Journal of Public Sector Management, 13(5), 417-446. http://dx.doi.org/10.1108/09513550010350832

Borges, T. B., \& Matias, M. A. (2011). Contabilidade gerencial no setor público: o caso de Minas Gerais. Contabilidade Vista \& Revista, 22(3), 73-98.

Brignall, S., \& Modell, S. (2000). An institutional perspective on performance measurement and management in the 'new public sector'. Management Accounting Research, 11(3), 281-306. http://dx.doi.org/10.1006/mare.2000.0136

Castaldelli, E., Jr., \& de Aquino, A. C. B. de (2011). Indicadores de desempenho em entidades fiscalizadoras superiores: o caso brasileiro. Contabilidade Vista \& Revista, 22(3), 15-40.

Christensen, M., \& Yoshimi, H. (2003). Public sector performance reporting: new public management and contingency theory insights. Government Auditing Review, 10, 71-83.

Donaldson, L. (2001). The contingency theory of organizations. Thousand Oaks, CA: Sage Publications.

Duarte, K., Lemos, C., Marini, C., \& Martins, H. F. (2006). Acordo de resultados. In R. Vilhena, H. F. Martins, C. Marini, \& T. B. Guimarães (Orgs.), O choque de gestão em Minas Gerais: políticas de gestão pública para o desenvolvimento (Cap. 6, pp. 95-127). Belo Horizonte: Editora UFMG.

Fryer, K., Antony, J., \& Ogden, S. (2009). Performance management in the public sector. International Journal of Public Sector Management, 22(6), 478-498. http://dx.doi.org/10.1108/09513550910982850

Gil, A. C. (2002). Como elaborar projetos de pesquisa (4a ed.). São Paulo: Atlas.

Gomes, E. G. M. (2009). Gestão por resultados e eficiência na administração pública: uma análise à luz da experiência de Minas Gerais (Tese de doutorado). Fundação Getúlio Vargas, Escola de Administração de Empresas de São Paulo, São Paulo, SP, Brasil. Recuperado de http://bibliotecadigital.fgv.br/dspace/bitstream/handle/10438/4652/72050100745.pdf?sequence=1

Grateron, I. R. G. (1999). Auditoria de gestão: utilização de indicadores de gestão no setor público. Caderno de Estudos, (21), 1-18. http://dx.doi.org/10.1590/S1413-92511999000200002

Guimarães, S. C. (2008). Avaliação de desempenho da gestão pública municipal (Dissertação de mestrado). Fundação Getúlio Vargas, Escola Brasileira de Administração Pública e de Empresas, Rio de Janeiro, RJ, Brasil. Recuperado de http://bibliotecadigital.fgv.br/dspace/handle/10438/3303

Holanda, A. N. C. (2003, outubro). Avaliação de políticas públicas: conceitos básicos, o caso do ProInfo e a experiência brasileira. Anais do Congreso Internacional del CLAD sobre la Reforma del Estado y de la Administración Pública, Panamá, 8.

Hood, C. (1991). A public management for all seasons? Public administration, 69(1), 3-19. http://dx.doi.org/10.1111/j.1467-9299.1991.tb00779.x

Hopwood, A. G. (1972). An empirical study of the role of accounting data in performance evaluation. Journal of Accounting Research, 10, 156-182. http://dx.doi.org/10.2307/2489870 
Jackson, A., \& Lapsley, I. (2003). The diffusion of accounting practices in the new "managerial" public sector. International Journal of Public Sector Management, 16(5), 359-372. http://dx.doi.org/10.1108/09513550310489304

Jannuzzi, P. D. M. (2002). Considerações sobre o uso, mau uso e abuso dos indicadores sociais na formulação e avaliação de políticas públicas municipais. Revista de Administração Pública, $36(1), 51-72$.

Jannuzzi, P. D. M. (2005). Indicadores para diagnóstico, monitoramento e avaliação de programas sociais no Brasil. Revista do Serviço Público Brasília, 56(2), 137-160.

Jensen, M. C., \& Meckling, W. H. (1976). Theory of the firm: managerial behavior, agency costs and ownership structure. Journal of Financial Economics, 3(4), 305-360. http://dx.doi.org/10.1016/0304-405X(76)90026-X

Johnsen, A. (2005). What does 25 years of experience tell us about the state of performance measurement in public policy and management? Public Money \& Management, 25(1), 9-17. http://dx.doi.org/10.1111/j.1467-9302.2005.00445.x

Julnes, P. D. L., \& Holzer, M. (2001). Promoting the utilization of performance measures in public organizations: an empirical study of factors affecting adoption and implementation. Public Administration Review, 61(6), 693-708. http://dx.doi.org/10.1111/0033-3352.00140

Kurunmaki, L., Lapsley, I., \& Melia, K. (2003). Accountingization v. legitimation: a comparative study of the use of accounting information in intensive care. Management Accounting Research, 14(2), 112-139. http://dx.doi.org/10.1016/S1044-5005(03)00019-2

Lapsley, I., \& Wright, E. (2004). The diffusion of management accounting innovations in the public sector: a research agenda. Management Accounting Research, 15(3), 355-374. http://dx.doi.org/10.1016/j.mar.2003.12.007

Lüder, K. G. (1992). A contingency model of governmental accounting innovations in the politicaladministrative environment. Research in Governmental and Nonprofit Accounting, 7, 99-127.

Malina, M. A., \& Selto, F. H. (2004). Choice and change of measures in performance measurement models. Management Accounting Research, 15(4), 441-469. http://dx.doi.org/10.1016/j.mar.2004.08.002

Malmi, T. (1999). Activity-based costing diffusion across organizations: an exploratory empirical analysis of Finnish firms. Accounting, Organizations and Society, 24(8), 649-672. http://dx.doi.org/10.1016/S0361-3682(99)00011-2

Manzini, E. J. (1990). A entrevista na pesquisa social. Didática, 26, 149-158.

Marques, K. C. M. (2012). Custeio alvo à luz da teoria da contingência e da nova sociologia institucional: estudo de caso sobre sua adoção, implementação e uso (Tese de doutorado). Universidade de São Paulo, Faculdade de Economia, Administração e Contabilidade, São Paulo, SP, Brasil. Recuperado de http://www.teses.usp.br/teses/disponiveis/12/12136/tde-29012013190708/pt-br.php

Naranjo-Gil, D. (2009). The influence of environmental and organizational factors on innovation adoptions: consequences for performance in public sector organizations. Technovation, 29(12), 810-818. http://dx.doi.org/10.1016/j.technovation.2009.07.003

Otley, D. T. (1980). The contingency theory of management accounting: achievement and prognosis. Accounting, Organizations and Society, 5(4), 413-428. http://dx.doi.org/10.1016/03613682(80)90040-9 
Przeworski, A. (1996). Nota sobre o estado e o mercado. Revista de Economia Política, 16(3), 115-120.

Queiroz, R. G. M., \& Ckagnazaroff, I. B. (2010). Inovação no setor público: uma análise do choque de gestão (2003-10) sob a ótica dos servidores e dos preceitos teóricos relacionados à inovação no setor público. Revista de Administração Pública, 44(3), 679-705. http://dx.doi.org/10.1590/S0034-76122010000300007

Scapens, R. W., \& Roberts, J. (1993). Accounting and control: a case study of resistance to accounting change. Management Accounting Research, 4(1), 1-32. http://dx.doi.org/10.1006/mare.1993.1001

Secretaria de Estado de Planejamento e Gestão. (2011). Plano mineiro de desenvolvimento integrado 2011-2030. Belo Horizonte, MG. Recuperado de http://www.planejamento.mg.gov.br/images/documentos/pmdi/pmdi_2011_2030.pdf

Silveira, M. C., \& Borges, T. B. (2008, novembro). A construção do alinhamento estratégico no estado de Minas Gerais. Anais do Encontro de Administração Pública e Governança, Salvador, BA, Brasil, 3.

Silveira, M. C., Fonseca, G. P. S., \& Oliveira, K. P. (2013). Os limites fiscais e orçamentários da reforma gerencial nos estados: o exemplo de Minas Gerais. Contabilidade Vista \& Revista, 23(1), $127-$ 163.

Simon, H. A. (1955). A behavioral model of rational choice. The Quarterly Journal of Economics, 69(1), 99-118. http://dx.doi.org/10.2307/1884852

Triviños, A. N. S. (1987). Introdução à pesquisa em ciências sociais: a pesquisa qualitativa em educação. São Paulo: Atlas.

Upping, P., \& Oliver, J. (2011). Accounting change model for the public sector: adapting luder's model for developing countries. International Review of Business Research Papers, 7(1), 364-380.

Williamson, O. E. (1985). The economic institutions of capitalism. New York, NY: The Free Press.

Wright, P., Kroll, M. J., \& Parnell, J. (2009). Administração estratégica: conceitos (C. A. Rimoli e L. R. Esteves, Trad.). São Paulo: Atlas. (Obra Original Publicada em 1998)

\title{
Dados dos Autores
}

\author{
Rosimeire Pimentel Gonzaga \\ Av. Antônio Carlos, 6627, Belo Horizonte, MG, Brasil. E-mail: ropgonzaga @ gmail.com \\ Fabio Frezatti \\ Av. Prof. Luciano Gualberto, 908, Cidade Universitária, 05508-010, São Paulo, SP, Brasil. E-mail: frezatti@ usp.br \\ Ivan Beck Ckagnazaroff \\ Av. Antônio Carlos, 6627, Belo Horizonte, MG, Brasil. E-mail: ivanbeck00@ gmail.com \\ Janilson Antônio da Silva Suzart \\ Esplanada dos Ministérios, Ed. Sede do Ministério da Fazenda, Bloco P, 70048-900, Brasília, DF, Brasil. E-mail: \\ jassuzart@yahoo.com.br
}

